\title{
Multidisciplinary approach to imaging for gender-affirming surgery: engaging surgeons, radiologists, and patients to ensure a positive imaging experience
}

\author{
Justin T. Stowell ${ }^{1}$, Vaz A. Zavaletta ${ }^{2}$, Evelyn F. Carroll ${ }^{3}$, Frances W. Grimstad ${ }^{4,5}$ \\ ${ }^{1}$ Department of Radiology, Mayo Clinic in Florida, Jacksonville, FL, USA; ${ }^{2}$ Department of Radiology, Division of Interventional Radiology, \\ Emory University School of Medicine, Atlanta, GA, USA; ${ }^{3}$ Department of Radiology, Mayo Clinic, Rochester, MN, USA; ${ }^{4}$ Division of Pediatric \\ and Adolescent Gynecology, Department of Surgery, Boston Children's Hospital, Boston, MA, USA; ${ }^{5}$ Department of Obstetrics, Gynecology and \\ Reproductive Biology, Harvard Medical School, Boston, MA, USA \\ Contributions: (I) Conception and design: All authors; (II) Administrative support: None; (III) Provision of study materials or patients: None; (IV) \\ Collection and assembly of data: JT Stowell; (V) Data analysis and interpretation: All authors; (VI) Manuscript writing: All authors; (VII) Final \\ approval of manuscript: All authors. \\ Correspondence to: Justin T. Stowell, MD. 4500 San Pablo Rd, Jacksonville, FL 32224, USA. Email: stowell.justin@mayo.edu.
}

\begin{abstract}
Medical imaging plays an integral role in the preoperative evaluation and postoperative management of transgender and gender diverse (TGD) patients who pursue gender-affirming surgery. Radiology department encounters can be a source of anxiety for patients of any demographic, including TGD patients. Although most imaging modalities are considered "non-invasive", certain imaging procedures and other aspects of the radiology encounter could be considered quite invasive to the TGD patient. The TGD patient may be worried that the imaging examination will have to address anatomy that they feel does not align with their gender identity, or reveal some abnormality or disheartening complication of their surgery. Simultaneously, the patient must also navigate potentially uncomfortable interactions with other patients in department waiting rooms, restrooms, and changing facilities as well as with radiology staff. As the referral source to imaging facilities, providers should advocate on behalf of their TGD patients. Referring providers should work with imaging facilities to ensure their patients will receive inclusive and affirming care and not be subject to discomfort on the part of gender identity or expression. Proactive and regular communication among radiology facilities, patients, and referring providers will ensure appropriate and sensitive care for this vulnerable population. A positive imaging experience can improve patient outcomes and the relationship between healthcare providers and the TGD community they serve.
\end{abstract}

Keywords: Radiology; mammography; urethrography; computed tomography; magnetic resonance imaging (MRI); ultrasound

Submitted Sep 15, 2020. Accepted for publication Jan 29, 2021.

doi: 10.21037/atm-20-6431

View this article at: http://dx.doi.org/10.21037/atm-20-6431

\section{Introduction}

Medical imaging plays an integral role in the preoperative evaluation and postoperative management of transgender and gender diverse (TGD) patients who pursue genderaffirming surgery. Referring providers may refer patients to radiology departments for various reasons ranging from routine pre-operative assessments (e.g., screening mammography prior to chest masculinization surgery in transmasculine patients) to post-operative assessment of suspected complications [e.g., fluoroscopy and contrastenhanced computed tomography (CT) to evaluate a neovaginal fistula after vaginoplasty]. TGD patients may also require image evaluation of general medical conditions.

Radiology department encounters can be a source 
of anxiety for patients of any demographic, but may be especially uncomfortable for TGD patients (1). Radiologic care is provided in a variety of practice settings ranging from mobile imaging units and outpatient imaging centers to stand-alone emergency departments and established specialty hospitals. Advanced imaging modalities make use of highly complex equipment such as magnetic resonance imaging (MRI) and interventional radiology procedure suites which may be intimidating for any patient. Although most imaging modalities are considered "non-invasive", certain imaging procedures and other aspects of the radiology encounter could be considered quite invasive to the TGD patient. The TGD patient may be worried that the imaging examination will have to address anatomy that they feel does not align with their gender identity, or reveal some abnormality or disheartening complication of their surgery. Simultaneously, the patient must also navigate potentially uncomfortable interactions with other patients in department waiting rooms, restrooms, and changing facilities as well as with radiology staff (including misgendering, deadnaming, and exposure of sensitive anatomy) during the performance of imaging procedures.

The needs and goals of one patient are not necessarily universal among all TGD persons. Therefore, proactive planning, communication, and partnership among referring providers, radiologists, and patients prior to referral of TGD patients for imaging can help to avoid these pitfalls and create an inclusive and affirming imaging environment. In this article, we provide a general framework for approaching imaging care for TGD patients that includes adaptive strategies for referring providers, radiology professionals, and patients to help to ensure a positive experience.

\section{Establish partnerships with inclusive imaging facilities}

Imaging facilities and the radiology professionals strive to deliver quality, patient-centered care and are guided and accredited by standards set forth by the American College of Radiology $(2,3)$. However, despite their best intentions, many radiology facilities and personnel may be unprepared to accommodate the unique needs of TGD patients $(1,4,5)$. Any aspect of an imaging encounter from scheduling to departure contributes to a TGD patient feeling welcomed or alienated. Table 1 provides a suggested checklist that referring providers may consider when assessing the inclusivity of an imaging facility.

\section{Scheduling and registration forms}

The scheduling and registration process, as the first point of contact, sets the tone for the facility's inclusion of TGD patients $(6,7)$. Registration should include opportunities to provide personal data in a discrete manner as well as include forms designed with gender-neutral language. Radiology facilities should review all intake and screening forms for opportunities to eliminate the use of gendered terminology (7). For example, when asking if a patient may be pregnant in the interest of radiation safety, these questions should avoid phrases such as "Are you a woman who may be pregnant?" replacing this instead with a more gender-neutral question such as "Are you or could you be pregnant?"

Additionally, intake forms should include data fields to appropriately ask sexual orientation and gender identity (SOGI) information. SOGI data collects vital demographics such as sex assigned at birth, gender identity, personal pronouns, chosen name, and legal name (6). For example, intake forms should include input fields for both gender identity and sex assigned at birth (so-called "two-step" gender identification). One survey of breast imaging facilities found that more than half (58\%) did not have intake forms that asked patients to provide their gender identity, and $55.9 \%$ did not assess gender identity at scheduling (4). Onequarter of facilities had female phrases auto-populated into the forms and into radiology reports (4). Another study found that $41 \%$ and $35 \%$ of TGD patients were not asked about personal pronouns or chosen name, respectively, and that in one-third of cases, radiology staff frequently used inappropriate pronouns in communication (1). Ten-percent of TGD patients reported challenges with insurance coverage for imaging services as a product of their gender identity (1). For example, a screening mammogram might be refused coverage by insurance for a transmasculine person whose legal gender may have been changed to male. To avoid insurance denials for imaging services, some TGD patients have been forced to supply only their sex assigned at birth on billing forms (1). Diligent collection of SOGI data not only will enable the best possible patient care, but also can be tracked for use in public health initiatives.

\section{Waiting rooms, changing rooms, and exam rooms}

The general environment of a facility such as waiting rooms, changing rooms, exam rooms, restrooms, and even general postings and décor can feel unwelcoming for TGD patients. Because of a communal history of discrimination 
Table 1 Checklist to help prepare for a positive imaging experience

General facility considerations

Patient intake process

Is there a place to convey sex assigned at birth, gender identity, chosen name and pronouns on intake forms?

Does the radiology facility have advanced notice that the patient is scheduled?

What insurance providers are accepted by the imaging facility?

Are there certain necessary documentation steps on behalf of the facility to ensure insurance coverage?

Can the imaging procedure be bundled with other procedures to minimize patient visits and exposure to uncomfortable situations?

Waiting and dressing rooms

Are the facility waiting rooms and exam rooms welcoming to LGBTQ persons (e.g., LGBTQ reading materials or postings, gender-neutral décor and color schemes, etc.)?

Are gender-neutral restrooms and changing rooms available?

Will un-robing be necessary?

Will patients need to be prepared to remove binding, tucking, or packing devices?

Policies

Does the facility have a non-discrimination policy that includes protections for LGBTQ patients? Is it provided to patients?

Staff interactions

Are imaging facility staff required to complete LGBTQ cultural sensitivity training that includes instruction on appropriate gender terminology?

Has staff been trained to consistently inquire with patients about pronoun use?

Does the imaging facility have a patient advocate or feedback system available to field complaints?

Procedural preparation

Is the imaging procedure sex-specific, and is there a need to clarify what anatomy is present, if the anatomy is native or constructed, and if the patient has chosen terms for their anatomy?

Does the patient experience distress regarding the anatomic region to be imaged?

What surgery, if any, has been performed?

Is the radiologist aware of the intended goals of the imaging study and expected imaging findings?

Has the procedure been explained to the patient in advance and have all questions been answered?

Are adaptations to the imaging protocol necessary to accommodate for an individual patient?

Is it necessary for the referring provider to speak with the radiologist in advance of complicated or atypical cases?

Does the referring provider need to be present to co-perform the procedure or co-interpret images with radiologist?

in health care settings, TGD patients often look for visible cues to indicate they are in a safe and inclusive space. Public postings such as the facility's nondiscrimination policy, diversity awards (e.g., Human Rights Campaign Health Equality Index), recognizable insignia (e.g., rainbow or transgender equality flags), or gender-neutral restroom signage may be silent indicators to the TGD patient that the imaging facility is open and inclusive $(1,6)$. Conversely, heavily gendered signage (e.g., "Women's Imaging") or décor schemes (e.g., pink wall paint or patient gowns) may make transmasculine and cisgender male patients feel uncomfortable.

\section{Interactions with bealth care staff and providers}

Direct interactions with radiology personnel including 
front desk staff, imaging technologists, nurses, advanced practice providers, and physicians contribute significantly to a patient's imaging experience. One-quarter $(24.5 \%)$ of TGD patients reported negative interactions with imaging staff with $32 \%$ needing to instruct imaging staff about TGD persons in order to receive appropriate care (1). In fact, $30 \%$ of TGD patients felt that imaging personnel seemed uncomfortable caring for a TGD patient, $22 \%$ felt that staff showed little concern for their comfort (1). The education of radiology personnel on culturally appropriate interactions with TGD patients is lacking across training curricula $(7,8)$. In one survey, fewer than $15 \%$ of breast imaging facilities offered sexual orientation and gender diversity training for their staff, and of those that did, only $33 \%$ made the training mandatory (4). Radiography educators felt that inclusion of TGD topics into curricula were moderately to extremely important, yet most reported they lacked sufficient knowledge to deliver the content (8). Unfortunately, the lack of education in TGD topics among radiology staff contributes to adverse encounters and substandard care.

\section{Collaborative solutions}

Care providers of TGD patients play a key role in educating and guiding their multi-disciplinary colleagues in the care of the pre- and post-operative TGD patient. As the referral source to imaging facilities, providers should advocate on behalf of their TGD patients. Providers may require and even offer to help arrange training for imaging center personnel on cultural sensitivity and appropriate interactions with TGD patients. Radiologists and referring providers should work collaboratively to adapt intake processes to incorporate these important changes.

Conversely, radiologists may request that providers who specialize in gender-affirming surgeries provide educational opportunities, such as presentations, guidebooks, and illustrations on the specific types of surgeries they perform as well as possible complications. In this way, radiologists can best collaborate with them to provide the most appropriate diagnostic imaging and image guided procedures. Radiologists and providers could work together to help train staff and technologists on specific goals of imaging exams and procedures that may require modified techniques and/or positioning.

Referring providers and radiologists could work together to ensure that appropriate diagnosis codes and indications are provided in documentation to potentially mitigate barriers to insurance coverage. Selective contract negotiations by imaging and hospital facilities with preferred inclusive insurance providers would also help ensure appropriate TGD patient care.

Referring providers should establish a network of preferred imaging centers that maintain sufficient standards for provision of culturally sensitive care of TGD patients. The best care of TGD patients will take place in an inclusive and affirming environment provided through multi-disciplinary collaboration and commitment.

\section{Procedures: modality selection and protocol tailoring}

Numerous imaging modalities are available to referring providers for assessment of patients before and after gender-affirming surgery. Selection of the most appropriate imaging study can be an overwhelming process (e.g., for suspected surgical complications). Close collaboration between providers, radiologists, and patients is invaluable to ensure the most appropriate imaging study is performed. The intended outcomes of each imaging examination are to maximize diagnostic utility while maintaining patient comfort and privacy during image acquisition. To achieve a balance for TGD patients, several imaging protocol adaptations may be required. A recent survey of 363 TGD patients found that image-guided procedures, ultrasound, and mammograms were the imaging examinations that most frequently caused unexpected physical and emotional discomfort among respondents (1). Nearly half of TGD patients who underwent ultrasound experienced emotional discomfort and nearly one-third experienced unexpected physical discomfort (1). Moreover, less than half (41.3\%) of respondents felt that the procedures were adequately explained to them beforehand and only one-quarter were encouraged to assist with the performance of the procedure (1). Table 2 summarizes several imaging modalities and potential protocol modifications that are may be utilized in the evaluation of various gender-affirming surgical complications.

\section{Imaging transfeminine patients}

Breast implant augmentation surgeries are considered safe with low complication rates (1.8\%) (10). Approximately $1.4 \%$ of transfeminine patients may require reoperation for postoperative hematoma evacuation or abscess drainage, and ultrasound may be used for diagnosis or image-guided 


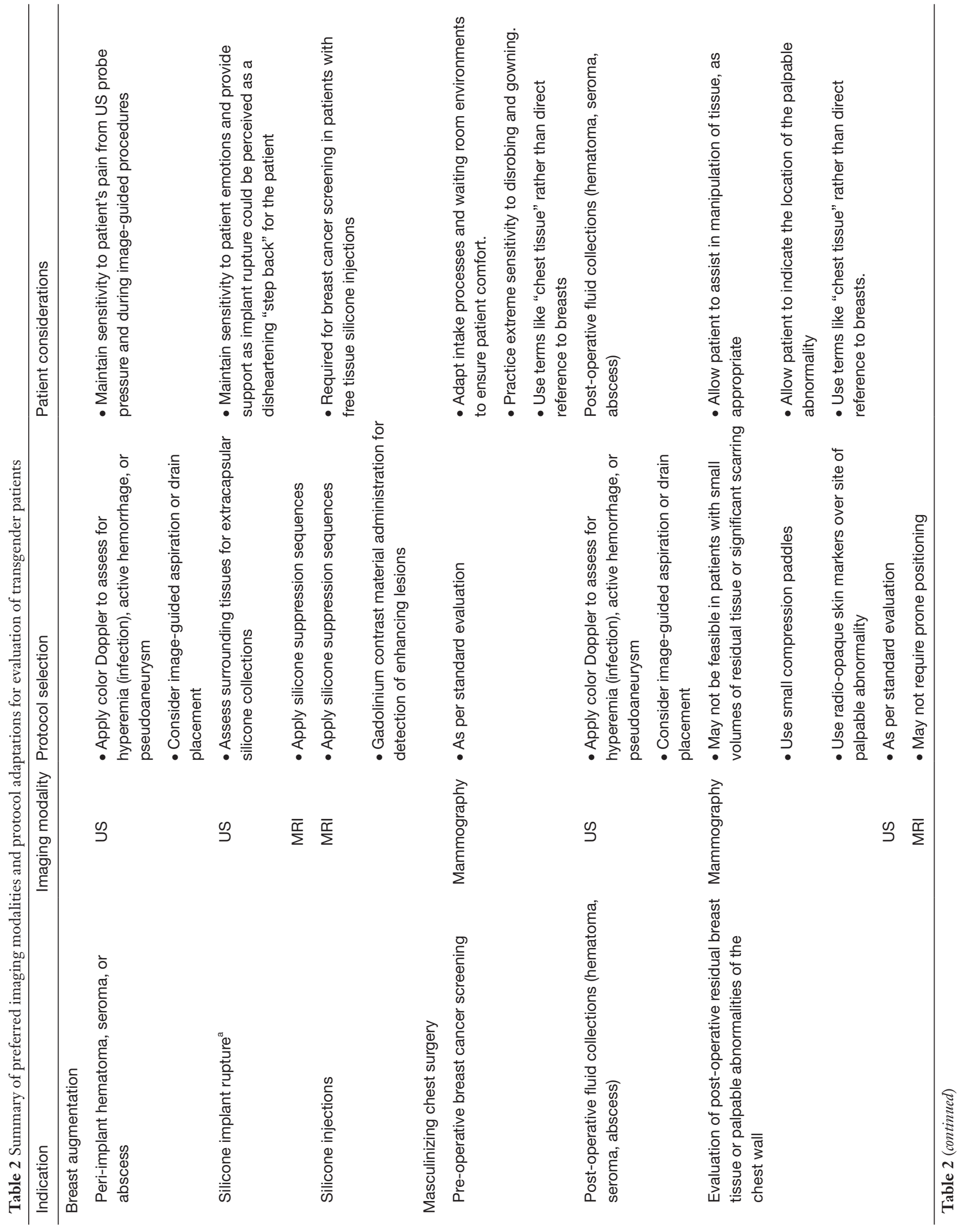




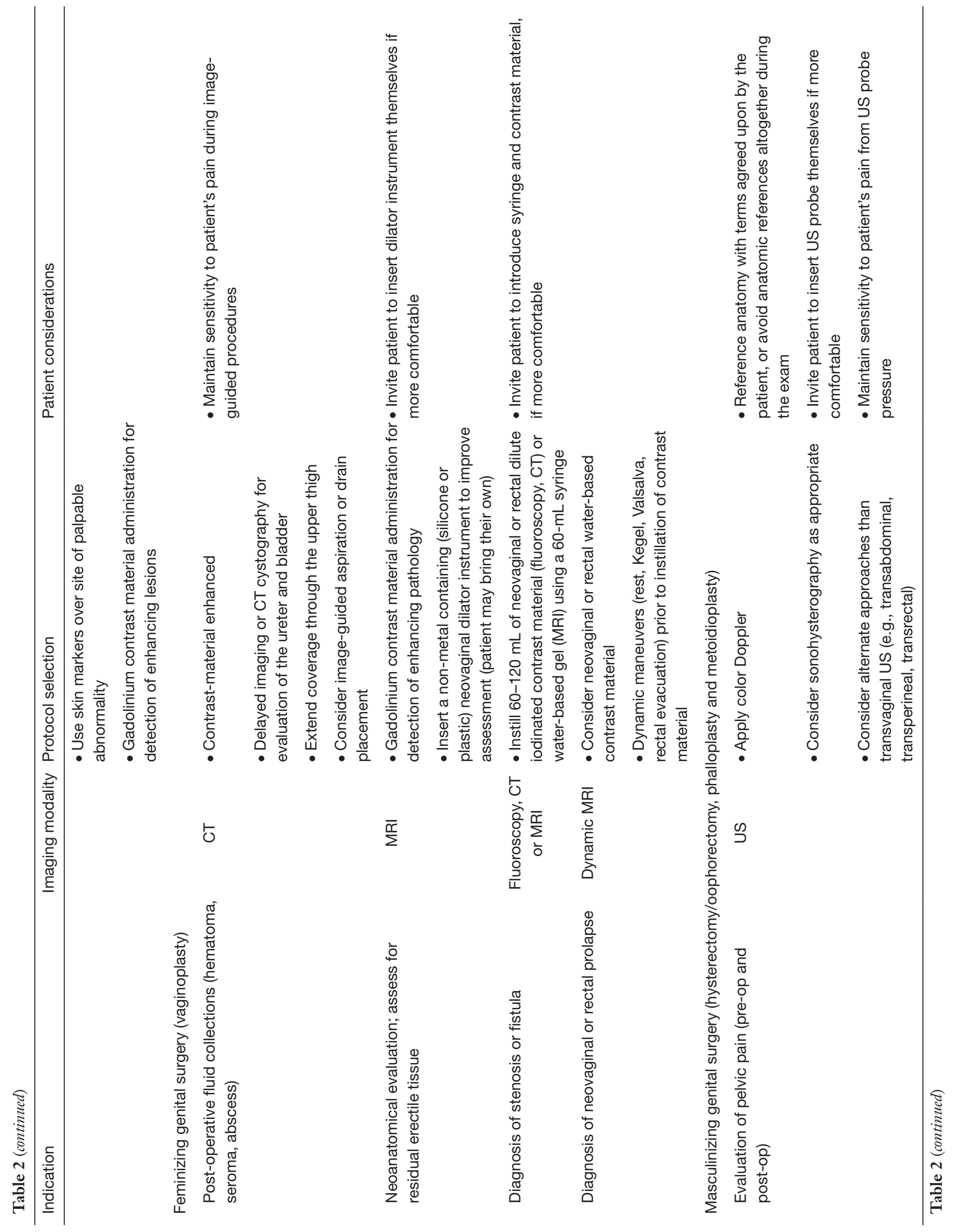




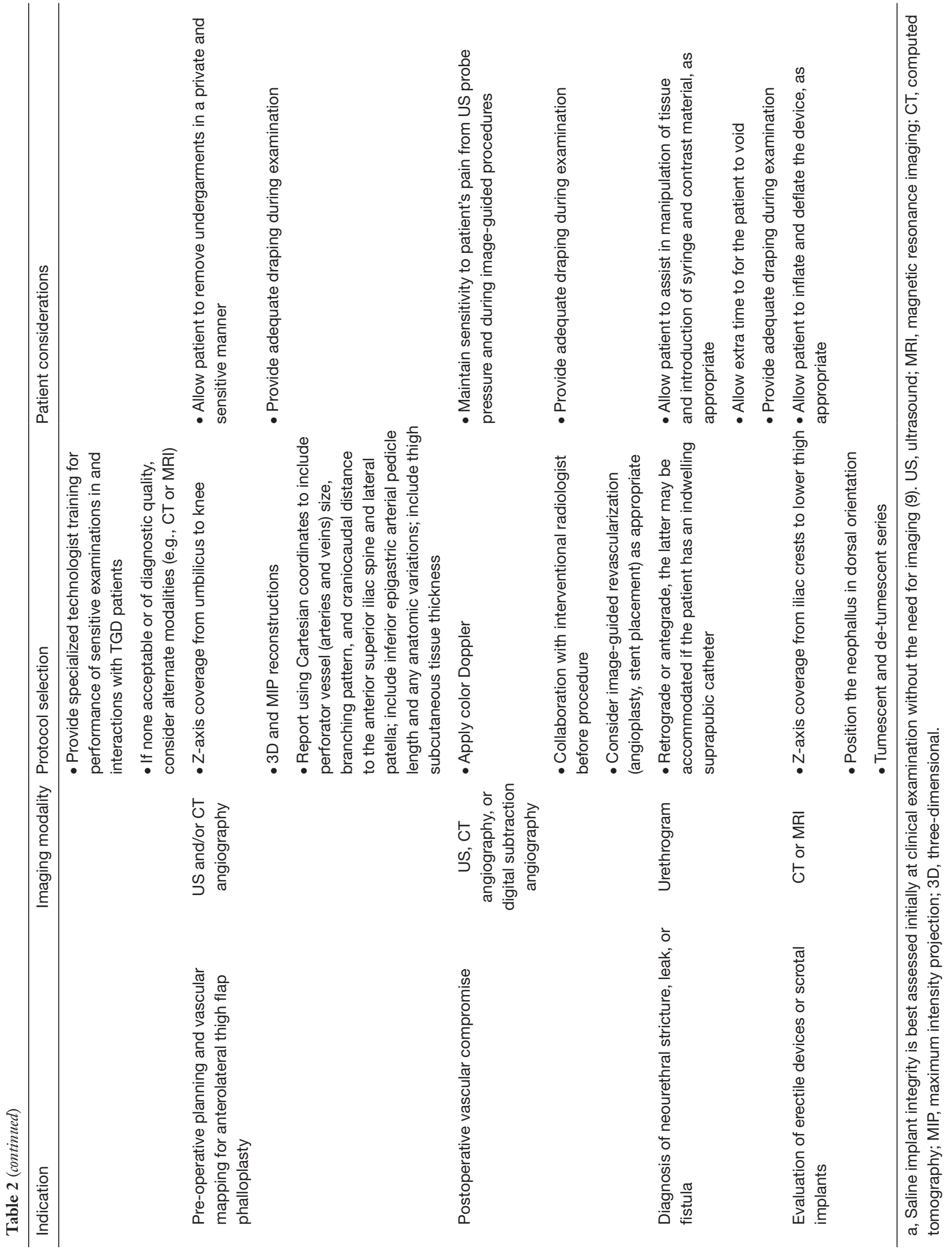


drain placement. MRI with silicone suppression sequences is the preferred modality for assessment of silicone breast implants (9). Patients who underwent augmentation with silicone injections require silicone-suppression breast MRI with gadolinium contrast material for detection of abnormal enhancement, preoperative planning when resection is planned, and for breast cancer screening (11).

Complications of vaginoplasty vary by surgical technique and the time from surgery with complications occurring in the first days to weeks usually related to wound complications or post-operative fluid collections (hematoma, abscess) (11). Incomplete resection of erectile tissue is a common source of hemorrhage after vaginoplasty. Contrastenhanced CT is a preferred imaging modality to assess for early complications after gender-affirming surgery. Delayed imaging or CT cystography may be useful to assess for bladder or ureteral injury. Complications that occur months to years after surgery include neovaginal stenosis, fistula, and prolapse. CT and MRI are the preferred modalities for assessing these complications, and protocol modifications such as introduction of a neovaginal dilator instrument or intracavitary contrast material can increase the sensitivity for detection of subtle abnormalities and better delineate their extent (11) (Table 2). To prevent stenosis, patients must practice routine dilation after vaginoplasty. To encourage the patient's assistance with dilator instrument placement or instillation of intracavitary contrast material might be less invasive for the patient and could prevent undue physical or psychologic trauma.

\section{Imaging transmasculine patients}

Although not explicitly included among the World Professional Association for Transgender Health (WPATH) Standards of Care requirements for chest masculinization surgery (12), referring providers should assess transmasculine patients' risk factors for breast cancer and refer eligible patients for screening mammography prior to chest masculinization surgery. Mammography with or without tomosynthesis is the recommended modality for breast cancer screening in patients with $<15 \%$ lifetime risk of breast cancer, and breast ultrasound or MRI may be appropriate for those with higher lifetime risk (13). Many transmasculine patients may be uncomfortable with the thought of undergoing mammography or other imaging procedures that might draw attention to their breast tissue. Practice Parameters for the performance of mammography and other breast imaging procedures have been established by the American College of Radiology to ensure reproducible image quality and diagnostic accuracy may prevent much modification to the technical aspects of image acquisition to accommodate transmasculine patients (14). However, referring providers may work with imaging facilities to ensure patients are comfortable and safe. For example, one may consider scheduling a transmasculine patient as a first or last appointment of the day to limit the need to encounter other patients at the facility. Additionally, an imaging facility might offer alternate changing areas or early rooming of a patient.

No formal, evidence-based standard has been developed for the need for and performance of breast cancer screening after chest masculinization surgery. Given the small volumes of chest wall tissue remaining after chest masculinization surgery, various technical adaptations may be required to perform mammograms. Small mammographic paddles may help to image residual tissue, but may be quite uncomfortable. Targeted diagnostic ultrasound is recommended for palpable abnormalities (15).

Transmasculine persons can develop idiopathic pelvic pain after the initiation of testosterone, and hysterectomy was found to be curative in $100 \%$ of patients (16). Up to $58 \%$ have persistent uterine bleeding $(16,17)$. Although higher rates of gynecologic cancers have not been demonstrated among transmasculine persons, pelvic ultrasound may be part of the diagnostic evaluation of pelvic pain and bleeding (18). A recent analysis found that transmasculine patients suffer high rates of emotional and physical discomfort during ultrasound examinations (1) indicating a need to adapt these exams or select alternate modalities when imaging transmasculine persons (Table 2).

Voiding cystourethography and retrograde urethography are frequently used for the evaluation of neourethral patency and integrity after perineal masculinization (phalloplasty, metoidioplasty) (19). Imaging facilities should schedule extra time for the urethrographic studies in transmasculine persons as patients may have heightened anxiety given that these procedures may require exposure of sensitive anatomic areas. Adequate draping of all patients with sheets or other coverings is paramount. Patients may experience voiding hesitancy or discomfort as this examination may be their first opportunity to void through the phalloplasty. Patients should be made comfortable and not rushed. Radiology personnel should be reduced to those necessary for the performance of the exam to protect privacy, and patients should be encouraged to assist with positioning and instillation of contrast material, as appropriate. 


\section{Empower the patient}

The World Health Organization defines patient empowerment as, "a process through which people gain greater control over decisions and actions affecting their health (20)." The American College of Radiology encourages a team approach to identify communication barriers and address patient concerns (21). A threeway dialogue among referring providers, radiologists, and patients ensures that the patient is embowered to understand both the rationale for an imaging examination and the results. Empowered patients guided by information and shared decision making develop mutual respect with their providers and confidence in the health system.

Having confidence in care providers and health systems is integral to patient empowerment. One in four TGD patients avoided medical care out of fear of being disrespected or mistreated (22). TGD patients have often had to navigate hostile and discriminatory healthcare environments and may come to our care feeling outcast and misunderstood (7). Patients should be made to feel welcome, included, and safe during imaging encounters. TGD patients reported that positive imaging encounters were most frequently associated with behaviors by radiology staff (1). These included being consistently treated with respect and dignity; being made to feel welcome, safe, and comfortable; and having their privacy protected during imaging encounters (1). An inclusive and affirming environment is necessary for patients to build confidence.

Radiologists and referring providers are responsible for providing information to their patients. This includes the purpose of the radiology examinations as well as the benefits and the risks within the context of the patient's surgery. Providers build trust with their patients when they provide anticipatory guidance about what to expect upon referral for imaging exams. To encourage TGD patients to ask questions about the imaging exam and to voice their concerns about various components of the imaging encounter builds mutual respect and allows for shared decision making. To empower a patient and provide a positive imaging experience can improve patient outcomes and the relationship between healthcare providers and the TGD community they serve.

\section{Conclusions}

Radiology professionals have an important role in the care provided to TGD people. Imaging facilities and examinations have the potential to incite additional trauma to TGD patients who are referred for imaging after genderaffirming surgery. Proactive and regular communication among radiology facilities, patients, and referring providers will ensure appropriate and sensitive care for this vulnerable population.

\section{Acknowledgments}

Funding: None.

\section{Footnote}

Provenance and Peer Review: This article was commissioned by the Guest Editors (Drs. Oscar J. Manrique, John A. Persing, and Xiaona Lu) for the series "Transgender Surgery" published in Annals of Translational Medicine. The article has undergone external peer review.

Conflicts of Interest: All authors have completed the ICMJE uniform disclosure form (available at http://dx.doi. org/10.21037/atm-20-6431). The series "Transgender Surgery" was commissioned by the editorial office without any funding or sponsorship. The authors have no other conflicts of interest to declare.

Ethical Statement: The authors are accountable for all aspects of the work in ensuring that questions related to the accuracy or integrity of any part of the work are appropriately investigated and resolved.

Open Access Statement: This is an Open Access article distributed in accordance with the Creative Commons Attribution-NonCommercial-NoDerivs 4.0 International License (CC BY-NC-ND 4.0), which permits the noncommercial replication and distribution of the article with the strict proviso that no changes or edits are made and the original work is properly cited (including links to both the formal publication through the relevant DOI and the license). See: https://creativecommons.org/licenses/by-nc-nd/4.0/.

\section{References}

1. Grimstad FW, Stowell JT, Gaddis M. Survey of Experiences of Transgender and Gender Nonbinary Patients During Imaging Encounters and Opportunities for Improvement. AJR Am J Roentgenol 2020;215:1136-42.

2. American College of Radiology (ACR) Accreditation. 
Accessed August 15, 2020. Available online: https://www. acr.org/Clinical-Resources/Accreditation

3. American College of Radiology (ACR) Commission on Patient- and Family-Centered Care. 2020. Accessed August 15, 2020. Available online: https://www.acr.org/ Member-Resources/Commissions-Committees/PFCC

4. Goldberg JE, Moy L, Rosenkrantz AB. Assessing Transgender Patient Care and Gender Inclusivity of Breast Imaging Facilities Across the United States. J Am Coll Radiol 2018;15:1164-72.

5. Huang SY, Zhang M, David M. Radiology's Engagement with Transgender Breast Imaging: Review of Radiology Practice Websites and Publications. J Breast Imaging 2020;2:147-51.

6. Kirkpatrick DL, Stowell J, Grimstad F, et al. Creating a Transgender-Inclusive Interventional Radiology Department. J Vasc Interv Radiol 2019;30:928-31.

7. Aarne Grossman VG. Providing Quality Care to the Transgender Patient in the Radiology Setting. J Radiol Nurs 2016;35:218-26.

8. Clark KR, Vealé BL. Assessing Transgender-Related Content in Radiography Programs in the United States: A Survey of Educators. J Med Imaging Radiat Sci 2018;49:414-9.

9. Lourenco AP, Moy L, Baron P, et al. American College of Radiology (ACR) Appropriateness Criteria: Breast Implant Evaluation. J Am Coll Radiol 2018;15:S13-25.

10. Cuccolo NG, Kang CO, Boskey ER, et al. Epidemiologic Characteristics and Postoperative Complications following Augmentation Mammaplasty: Comparison of Transgender and Cisgender Females. Plast Reconstr Surg Glob Open 2019;7:e2461.

11. Stowell JT, Grimstad FW, Kirkpatrick DL, et al. Imaging Findings in Transgender Patients after Gender-affirming Surgery. Radiographics 2019;39:1368-92.

12. Coleman E, Bockting W, Botzer M, et al. Standards of Care for the Health of Transsexual, Transgender, and Gender-Nonconforming People, Version 7. Int J Transgend 2012;13:165-232.

13. Mainiero MB, Moy L, Baron P, et al. American College of Radiology (ACR) Appropriateness Criteria: Breast Cancer Screening. J Am Coll Radiol 2017;14:S383-90.

Cite this article as: Stowell JT, Zavaletta VA, Carroll EF, Grimstad FW. Multidisciplinary approach to imaging for gender-affirming surgery: engaging surgeons, radiologists, and patients to ensure a positive imaging experience. Ann Transl Med 2021;9(7):610. doi: 10.21037/atm-20-6431
14. American College of Radiology (ACR) Practice Parameter for the Performance of Screening and Diagnostic Mammography. 2018. Available online: https://www.acr. org/-/media/ACR/Files/Practice-Parameters/ScreenDiag-Mammo.pdf

15. Heller SL, Lourenco AP, Niell BL, et al. American College of Radiology (ACR) Appropriateness Criteria: Imaging after Mastectomy and Breast Reconstruction. 2020. Accessed August 15, 2020. Available online: https://acsearch.acr.org/docs/3155410/ Narrative/?_ga $=2.254775692 .527287507 .1599488982$ 1569866093.1572310536

16. Grimstad FW, Boskey E, Grey M. New-Onset Abdominopelvic Pain After Initiation of Testosterone Therapy Among Trans-Masculine Persons: A CommunityBased Exploratory Survey. LGBT Health 2020;7:248-53.

17. Grimstad FW, Fowler KG, New EP, et al. Uterine pathology in transmasculine persons on testosterone: a retrospective multicenter case series. Am J Obstet Gynecol 2019;220:257.e1-7.

18. Expert Panel on GYN and OB Imaging, Robbins JB, Sadowski EA, et al. ACR Appropriateness Criteria ${ }^{\circledR}$ Abnormal Uterine Bleeding. J Am Coll Radiol 2020;17:S336-45.

19. Dabela-Biketi A, Mawad K, Li H, et al. Urethrographic Evaluation of Anatomic Findings and Complications after Perineal Masculinization and Phalloplasty in Transgender Patients. Radiographics 2020;40:393-402.

20. WHO Guidelines on Hand Hygiene in Health Care: First Global Patient Safety Challenge Clean Care Is Safer Care. In: Patient empowerment and health care. World Health Organization, Geneva. 2009. Accessed September 7, 2020. Available online: https://www.ncbi.nlm.nih.gov/books/ NBK144022/

21. American Institute for Radiologic Pathology (AIRP) Newsletter: Empowering Patients. 2015. Accessed September 7, 2020. Available online: https://www.airp.org/ Newsletter/Empowering-Patients

22. James SE, Herman JL, Rankin S, et al. The Report of the 2015 U.S. Transgender Survey. Washington, DC: National Center for Transgender Equality. National Center for Transgender Equality, 2016. 